\title{
Cerebral infarction complicating intravenous immunoglobulin therapy in a patient with Miller Fisher syndrome
}

\author{
Benjamin Turner, Adrian J Wills
}

\begin{abstract}
Intravenous immunoglobulin (IVIg) therapy is being increasingly used in a wide range of neurological conditions. However, treatment is expensive and side effects may be severe. A patient with Miller Fisher syndrome who developed cortical blindness as a consequence of occipital infarction precipitated by IVIg is reported on.

(F Neurol Neurosurg Psychiatry 2000;68:790-791)
\end{abstract}

Keywords: Miller Fisher syndrome; intravenous immunoglobulin; cerebral infarction

\section{Case report}

A 60 year old woman presented with a 5 day history of left sided ptosis, diplopia, paraesthesia of the hands and feet, and impaired manual dexterity. One week previously she had had a diarrhoeal illness lasting 24 hours.

On examination she was apyrexial and mildly tachycardic. Her forced vital lung capacity was 1.45 1. Examination of the cranial nerves showed a left sided ptosis with a total external ophthalmoplegia of the left eye and impaired abduction of the right eye. The pupillary reflexes were normal to light and accommodation. She had a bulbar palsy causing dysarthria and mild dysphagia with bilateral facial weakness. Examination of the peripheral nervous system showed mild (4/5 MRC scale) weakness in the arms and legs associated with loss of deep tendon reflexes. There was severe loss of proprioception and diminished vibration sense in the hands and feet with associated limb ataxia yet pain and temperature sensation was relatively preserved. She was unable to walk.

It was considered that she had the Miller Fisher variant of Guillain-Barré syndrome complicating a campylobacter jejuni infection 1 week earlier. Subsequent investigations disclosed raised IgG antiganglioside antibodies to GQ1b glycolipids (titre of 1/3000) and high $\operatorname{IgM}(+1280)$ and $\operatorname{IgA}(+320)$ titres against campylobacter jejuni. There was neurophysiological evidence of an axonal sensory neuropathy (sensory conduction in the left sural and radial nerves was absent; left median motor compound muscle action potential was $7.2 \mathrm{mV}$ with a conduction velocity of 43.4 $\mathrm{m} / \mathrm{s})$. Electrolytes and brain CT performed at the referring hospital were normal. A lumbar puncture was performed on day 5 of the illness and demonstrated normal CSF constituents with a protein concentration of $0.1 \mathrm{~g} / \mathrm{l}$. Intravenous immunoglobulin (IVIg) was given for 5 days at a dose of $0.4 \mathrm{~g} / \mathrm{kg} /$ day starting 6 days after the onset of neurological symptoms.

On the second day of IVIg treatment it was noted that the plasma sodium had fallen to 128 $\mathrm{mmol} / \mathrm{l}$, with a urinary sodium of $205 \mathrm{mmol} / \mathrm{l}$, random cortisol of $510 \mathrm{nmol} / 1$, and normal renal function, fulfilling the criteria for the syndrome of inappropriate antidiuretic hormone (SIADH). Five days later the plasma sodium reached $124 \mathrm{mmol} / \mathrm{l}$ and a $1.5 \mathrm{l} /$ day fluid restriction was instigated. Her neurological status began to improve but after 3 days she became acutely confused. Within a few hours her confusion had settled but she complained of visual loss. Examination showed that she had perception of light only in both eyes with normal pupillary responses. Magnetic resonance imaging showed bilateral occipital lobe infarcts (figure). Plasma viscosity was measured at 1.85

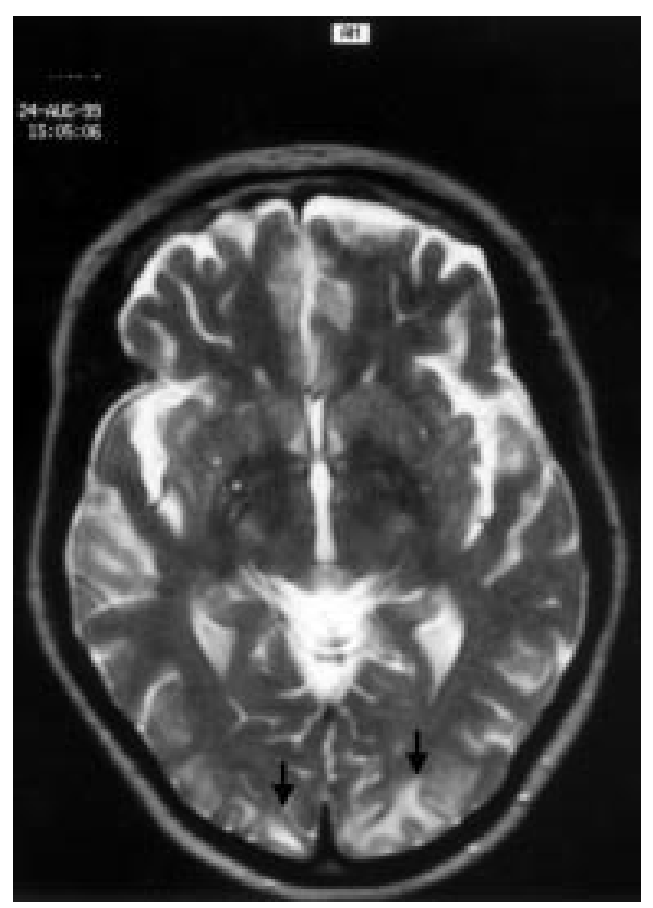

Magnetic resonance image showing bilateral occipital infarction (arrows). 
centipoise (cp) and over the next 3 days rose to $2.01 \mathrm{cp}$ (normal range $1.5-1.72$ ). Subsequent magnetic resonance angiography of the cerebral blood vessels and transoesophageal echocardiography showed no evidence of an embolic source. There was no evidence of previous hypotension or any episodes of arrhythmia.

By the time of discharge the patients' vision had improved to $6 / 9$ bilaterally. Her left eye remained moderately paretic and her right eye was normal. The left sided ptosis and facial weakness had improved and bulbar function was normal. She was able to walk unaided and had normal power in her limbs but remained arreflexic. Romberg's test was negative and joint position sense had normalised.

\section{Discussion}

Guillain-Barré syndrome is an acute monophasic peripheral neuropathy characterised by generalised muscle weakness and has an incidence of 1-2/100 000/year and a mortality of $10 \%{ }^{1}$ In 1956 Fisher described three patients with a syndrome consisting of ophthalmoplegia, ataxia, and areflexia. This is considered a variant of Guillain-Barré syndrome. About $6 \%$ of patients with Guillain-Barré syndrome start as Miller Fisher syndrome, half of which go on to develop marked weakness and half remain pure Miller Fisher syndrome. In Miller Fisher syndrome the external ophthalmoplegia usually begins symmetrically with abduction affected initially. Ptosis, bilateral but asymmetric, is present in over half the patients and facial and bulbar palsy does occur. ${ }^{2}$ The ataxia may involve the limbs or gait equally often with clumsiness of fine finger movements; however, loss of joint position sense is not invariable. Most patients have a preceding illness and this is often Campylobacter jejuni enteritis. ${ }^{3}$ It is thought that these postinfectious syndromes are caused by the production of antibodies to gangliosides, which are substantial components of neuronal membranes and have regulatory roles. ${ }^{4}$ These antibodies may arise due to molecular mimicry between Campylobacter jejuni lipopolysaccharides and gangliosides. ${ }^{3}$ In $90 \%$ of patients with Miller Fisher syndrome there are high titres of antibodies to the GQ1b ganglioside. ${ }^{5}$

In clinical practice the aetiology of SIADH is often unclear but it is recognised in association with Guillain-Barré syndrome. The diagnosis of SIADH requires hyponatraemia with low plasma osmolality, increased urinary sodium excretion, absence of volume depletion, and normal renal, adrenal, and hepatic function. Symptoms of hyponatraemia range from a mild confusion, clouding of consciousness to coma, and seizures. ${ }^{6}$ In our patient the acute onset of loss of vision and short lived confusion are more consistent with acute ischaemia of the occipital lobe than "hyponatraemic encephalopathy".

Intravenous immunoglobulin is being used to treat an increasing number of neurological illnesses including Guillain-Barré syndrome, multiple sclerosis, chronic inflammatory demyelinating polyneuropathy, myaesthenia gravis, dermatomyositis, and multifocal motor neuropathy with conduction block. Immunoglobulin treatment has been quoted as having a minor complication rate of $59 \%$ and $4.5 \%$ of patients have serious complications including neutropenia, congestive heart failure, and renal failure. $^{7}$ There have been three previously reported cases of cerebral infarction after IVIg, and cerebral vasospasm, cerebral vasculitis, and serum hyperviscosity have been implicated in the pathogenesis. ${ }^{8}$ In our patient cerebral infarction may have arisen as a consequence of IVIg related hyperviscosity exacerbated by fluid restriction. All patients receiving IVIg must have careful fluid balance management and if SIADH occurs fluid restriction should only be instigated when hyponatraemic symptoms are severe. ${ }^{6}$ In view of the risk of serious adverse events in those patients receiving IVIg, the treatment must be clearly justified.

1. Hughes RAC. Intravenous IgG in Guillain-Barré syndrome. BMF 1996;313:376-77.

2 Ropper AH. Miller Fisher syndrome and other acute variants of Guillain-Barré syndrome. In: Mcleod JG, ed. Inflammatory neuropathies. London: Baillière's Clinical Neurology 1994;95-106.

3 Yuki N, Taki T, Takahashi M, et al. Molecular mimicry between GQ1b ganglioside and liposaccharides of Campylobacter jejuni isolated from patients with Fishers syndrome. Ann Neurol 1994;36:791-3.

4 Ledeen RW, Wu G. Ganglioside function in the neuron. Trends in Glycoscience and Glycotechnology 1992;4:174-87.

5 Plomp JJ, Molenaar PC, O'Hanlon GM, et al. Miller-Fisher anti-GQ1b antibodies: $\alpha$-latrotoxin-like effects on motor end plates. Ann Neurol 1999;45:189-99.

6 Gill G, Leese G. Hyponatraemia: biochemical and clinical Gill G, Leese G. Hyponatraemia: biochemical
perspectives. Postgrad Med f 1998;74:516-23.

perspectives. Postgrad Med 7 1998;74:516-23.
7 Wills AJ, Unsworth DJ. A practical approach to the use of Wills AJ, Unsworth DJ. A practical approach to the use of
intravenous immunoglobulin in neurological disease. Eur intravenous immun

8 Stangel M, Hartung HP, Marx P, et al. Intravenous immunoglobulin treatment of neurological autoimmune diseases. F Neurol Sci 1998;153:203-14. 